\title{
COVID-19 under 19: A Meta-analysis
}

\author{
Nagham Toba ${ }^{1}$, Shreya Gupta ${ }^{2}$, Abdulrahman Ali², Mariam ElSaban ${ }^{3}$, Amar HK², Samuel \\ $\mathrm{Ho}^{2}$, and Rizwana Popatia ${ }^{2}$ \\ ${ }^{1}$ Mohammed Bin Rashid University of Medicine and Health Sciences College of Medicine \\ ${ }^{2}$ Mohammed Bin Rashid University of Medicine and Health Sciences \\ ${ }^{3}$ Mohammed Bin Rashid University of Medicine and Health Sciences Hamdan Bin \\ Mohammed College of Dental Medicine
}

August 23, 2020

\begin{abstract}
Background: The COVID-19 pandemic has caused a global havoc with our limited understanding of the SARS-CoV-2, disease manifestations and management. Inadequacy of available data in pediatric patients coupled with evolving disease course makes it imperative to conduct a meta-analysis assessing the results of pediatric COVID-19 studies over the course of the pandemic. Methods: A random-effect meta-analysis was conducted using PRISMA guidelines. Two databases were screened for pediatric COVID-19 studies and selected articles reviewed for demographic, co-morbidities, clinical manifestations, laboratory and radiological evaluation, treatment and outcomes. Prevalence with mean and $95 \%$ confidence interval was calculated. Results: Out of 1703 articles, 37 articles comprising of 993 patients for a period of over six months and 72 variables were selected. This meta-analysis revealed that one-fourth of patients were asymptomatic (23.8\%, 95\% CI 17.6-31.2\%) Fever (52.5\%, 95\% CI 45.7$59.1 \%)$ and cough $(47.6 \%, 95 \%$ CI $41.2-54.0 \%)$ were the most common symptoms. The most frequently encountered white blood count abnormalities were neutropenia (16.6\%, 95\% CI 10.2-25.8\%), lymphocytosis (15.3\%, 95\% CI 9.9-23.0\%) and leukopenia $(13.9 \%, 95 \%$ CI 10.1-18.8\%). Ground glass opacities were the most common radiological finding of children with COVID-19 (35.9\%, 95\% CI $29.4-43 \%)$. The hospitalization rate was $95.9 \%$ (95\% CI 91.9-98.0\%) of which $11.2 \%$ (95\% CI $4.7-24.2)$ were ICU admissions, and 4.2\% (95\% CI 2.6-6.9\%) died. Conclusion: Majority of pediatric patients are asymptomatic or have mild manifestations similar to other upper respiratory viruses. Serious disease and death occurred in $15.4 \%$. More studies are needed from a wider geographic area as the pandemic continues.
\end{abstract}

\section{Introduction}

The novel coronavirus (COVID-19) pandemic, caused by severe acute respiratory syndrome coronavirus 2 (SARS-CoV-2) ${ }^{1}$, has created a global healthcare pandemonium with over 21 million cases and over 755,000 deaths reported worldwide as of early August $2020^{2}$. Since its inception in Wuhan, China in December 2019 as a cluster of cases presenting with influenza-like illness, the virus' uncurbed spread has spanned over 213 countries and territories resulting in the World Health Organization (WHO) announcing it as a pandemic on 11 March $2020^{3}$. The disease presented itself in the earlier stages primarily as a respiratory illness with higher morbidity and mortality in older individuals ${ }^{4}$. However, the evolving trends of this novel disease highlighted the diversity of presenting features and involvement within pediatric age groups. To date, most of the available literature focuses on the adult population leaving a noticeable gap in description of pediatric COVID-19. Of assurance, COVID-19 has fared well in children with initial trends showing milder form of illness, less hospitalizations and minimal fatality as reported in various studies worldwide ${ }^{5-7}$. However, the evolving disease trends have depicted varied severity in children with United States Center for Disease 
Control (CDC) releasing a health advisory reporting a Multisystem Inflammatory Syndrome in Children (MIS-C) related to COVID-19 on May $14^{\text {th }}, 2020^{8}$. Our progressing knowledge about the disease in the past few months necessitates a data-rich meta-analysis of pediatric COVID-19 to establish statistical significance across these studies and thereby, understanding the validity of the observed parameters.

The study aims to describe clinical presentation, laboratory and radiographic findings, treatment modalities and outcomes of pediatric patients under age 19 with COVID-19. Furthermore, the perpetual rapid escalation of cases worldwide and upcoming re-opening of educational institutes necessitates a more inclusive look into pediatric presentations of COVID-19 to guide immediate health and education policy-making worldwide.

\section{Methodology}

\section{Protocol}

The study protocol was based on the Preferred Reporting Items for Systematic Reviews and Meta-Analyses (PRISMA) guidelines and was reported using the PRISMA checklist ${ }^{9}$. The protocol was registered on the International Prospective Register of Systematic Reviews (PROSPERO) with the registration number CRD42020186160 on 17 May 2020.

\section{Literature Search and Data Extraction}

The databases PubMed and Scopus were reviewed from December 1, 2019 to July 3, 2020, to identify all relevant COVID-19 primary publications. The keywords and Medical Subject Headings (MeSH) terms selected included"Novel coronavirus 2019", "COVID-19" and "SARS-CoV-2", and the target population was specified with the terms "pediatric", "children", "infant", "neonate", "adolescent". The last search was performed on July 3rd, 2020 and the search was not limited by language (translation performed with Google Translate) or geographic region.

\section{Eligibility criteria and Study Selection}

Inclusion criteria for screening

Study selection methodology entailed initially screening articles using title and abstract and subsequent fulltext screening. All available peer-reviewed original articles (case reports, case series, cohort studies, crosssectional studies etc.) pertaining to pediatric COVID-19 published in the literature in the aforementioned time frame were included in this study. Selected articles must have subjects with SARS-CoV-2 infection confirmed via real-time reverse transcriptase polymerase chain reaction (RT-PCR) using upper respiratory swabs. The pediatric population comprised of ages 0-18 years (including neonates). The selected articles included variables on demography, risk factors, clinical manifestations, laboratory and radiological findings, treatment, and outcomes.

Articles were excluded due to the inability to extract pediatric data from adult data separately. The publication types excluded were review articles and studies such as letters, correspondences or comments that had no extractable primary pediatric data. Articles with irrelevant clinical study focus (e.g. epidemiology, modeling, animal data, post-mortem data) and non-clinical study focus (e.g. genetics, diagnostic techniques or virology) were excluded as well.

\section{Inclusion criteria for Meta-analysis data}

Studies that qualified for initial screening for data extraction, as mentioned above, were further filtered with stringent criteria. The inclusion criteria were optimized for selection of articles with sufficient data and sample size for data synthesis.

\section{Data Extraction and Quality Assessment}

Four investigators worked on title/abstract, full-text screening and data extraction in pairs (AA and ME, SG and NT) on a shared data extraction form. Any disagreements were resolved by consulting one of the investigators from the other pair. The investigators extracted data on demographics, co-morbidities, signs 
and symptoms, laboratory investigations, radiological investigations, treatment and outcomes of COVID-19 in pediatric patients. The Joanna-Briggs Institute (JBI) checklists were utilized for the critical appraisal of case series and cross-sectional studies ${ }^{10}$. The investigators assigned 2 points for 'Yes', 1 point for 'Unclear' and 0 points for 'No/Inapplicable'. The average score of the 2 investigators generated the final JBI score. Each checklist had a different cumulative score which was scaled out of 10 (Table 1). A score $>7$ reflected a high-quality study, 5-7 moderate-quality and $<5$ a low-quality study.

\section{Statistical Analysis}

Percentages were calculated to describe the distribution of the categorical dichotomous variables. For continuous data, the pooled prevalence with mean and $95 \%$ confidence intervals (CI) were calculated. For studies reporting the mean with $95 \% \mathrm{CI}$ or the range of the data, the formula, (upper limit-lower limit)/4, was used to extract the standard deviation (SD).

The meta-analysis was conducted on Comprehensive Meta-Analysis version 3.3.070 software. The randomeffect model was implemented to estimate the pooled prevalence and 95\% CI. Pooled percentage, proportion and corresponding $95 \% \mathrm{CI}$ were calculated to summarize the weighted effect size for all binary variables. The measure of heterogeneity reported included the Cochran's Q statistics, $\mathrm{I}^{2}$ index with the level of heterogeneity defined as low $<25$, moderate $>50$, and high $>75$, and the tau square $\left(\mathrm{T}^{2}\right)$ test. Publication bias was assessed with a funnel plot and Egger's test.

\section{Results}

\section{FIGURE 1}

As shown by the literature retrieval flowchart in Figure 1, 1703 pediatric COVID-19 articles were searched from two databases (PubMed and Scopus) from December 2019 to July 2020 with a predefined search strategy. Out of those, 734 duplicate studies were excluded and 784 studies did not meet the eligibility criteria for meta-analysis, owing to inappropriate study type/ focus or lack of relevant clinical pediatric data when screened with title and abstract. Of the remaining 185 full text articles that met the eligibility criteria, 37 studies comprising of 993 patients sieved through the rigorous criteria of inclusion for meta-analysis. Remaining studies were excluded due to inadequate sample size, insufficient availability of data and other reasons described in Figure 1. The journal name, PMID and characteristics of the 37 studies selected for meta-analysis, and funnel plots and forest plots are shown in the supplementary material.

\section{TABLE 1}

Table 1 lists the study characteristics of the 37 articles selected for meta-analysis with their author information, date of publication, country of origin, study design, number of pediatric patients and JBI scaled score. As shown in the table, these studies were published between March and July 2020. Majority (84\%) of the studies were from China, whereas the rest originated from Turkey, France, UK, and Switzerland. Case series comprised of more than half $(60 \%)$ of the study design and the remaining half were cross-sectional. Half of the articles had sample size of 2-10 patients and about $15 \%$ had a sample size of over 40 subjects. The mean JBI scaled score is 8 ( $0.88 \mathrm{SD})$ out of 10 . Approximately $90 \%$ of the selected articles yielded a JBI scaled score of [?]7, affirming good quality of the selected articles.

\section{Meta-Analysis Results}

\section{TABLE 2}

\section{Demographic Characteristics}

Of the 993 pediatric patients analyzed, the mean age of the patients across the studies was 6.63 years (SD: 0.9; 95\% CI, 4.9-8.4). Males comprised 52.2\% (95\% CI, 47.7 - 56.6\%) of the population (Table 2).

Co-morbidities 
The total prevalence of co-morbidities associated with pediatric COVID-19 based on 18 studies was $14.5 \%$ (95\% CI, $8.3-24.2 \%)$. The three most common co-morbidities were history of congenital heart disease $5 \%$ (95\% CI, $2.5-9.8 \%)$, obesity $4.9 \%$ (95\% CI, $1.9-11.8 \%)$ and prematurity $4 \%$ (95\% CI, $1.5-10.4 \%$ ).

Clinical Presentation

It was notably interesting that approximately one-fourth of the patients were asymptomatic $(23.8 \%$ with $95 \%$ CI, 17.6-31.2\%). Amongst the symptomatic, fever (52.5\% with $95 \%$ CI, $45.7-59.1 \%)$ and cough $(47.6 \%$ with $95 \%$ CI, $41.2-54.0 \%$ ) were the most common symptoms. Expectoration was a notable symptom in 19.5\% (95\% CI, 11.7 - 30.7). The spectrum of clinical manifestations included generalized (headache, fatigue, rash and myalgia), respiratory (cough, dyspnea, rhinorrhea, nasal congestion and sore throat) and gastrointestinal (nausea, vomiting, abdominal pain and diarrhea) symptoms.

\section{TABLE 3}

\section{TABLE 4}

Laboratory Investigations

The most commonly encountered white blood cell (WBC) abnormalities were neutropenia, lymphocytosis and leukopenia that were present in $16.6 \%$ (95\% CI 10.2-25.8\%), $15.3 \%$ (95\% CI $9.9-23.0 \%$ ) and $13.9 \%$ (95\% CI $10.1-18.8 \%)$ patients respectively. From the array of abnormal laboratory findings in pediatric SARS-CoV-2 infection, more prevalent ones included elevated inflammatory markers such as C-reactive protein (CRP) (elevated in 22.6\%, 95\% CI 17.2-29\%) and procalcitonin (elevated in $31.8 \%, 95 \%$ CI $22.3-$ $\left.43.1, \mathrm{I}^{2}>75\right)$, abnormal liver function tests (LFT) $\left(21.3 \%, 95 \%\right.$ CI $\left.14.6-30.0 \%, \mathrm{I}^{2}>75\right)$, creatinine kinase (CK) (elevated in 17\%, 95\% CI 10.1 - 27.3), D-dimer (elevated in 16.2\%, 95\% CI 10.9-23.4\%) and cardiac biomarkers troponin (high in $23 \%, 95 \%$ CI $3.6-70.0 \%, \mathrm{I}^{2}>75$ ) and creatinine kinase - MB (CK-MB) (high in $21.1 \%, 95 \%$ CI $\left.11.9-34.6 \%, \mathrm{I}^{2}>75\right)$. An additional interesting finding extracted from 21 studies showed co-infections $(22.4 \%, 95 \%$ CI $15.0-31.9 \%)$ in children with COVID-19, the most common being bacterial co-infections (19.9\% 14.3-26.9\%) followed by influenza A or B $(6.8 \%, 95 \%$ CI, $3.2-13.7 \%)$.

Radiological Findings

Computed Tomography (CT) of the chest appeared to be the imaging modality of choice over chest x-rays from the meta-analyzed studies in Table 3 . The most common CT abnormality, occurring in more than one third of the patients, was ground glass opacities (GGO) (35.9\% with 95\% CI 29.4 - 43\%).

Treatment

93.9\% of patients (95\% CI $87.6-97.1 \%$ ) received some form of treatment (includes the treatment options listed in Table 4 as well as symptomatic and herbal treatments). Antiviral therapies were the most prescribed treatments at $82.7 \%(95 \%$ CI $62.6-93.2 \%)$ and included interferon $\alpha$, lopinavir/ritonavir, oseltamivir and, umifenovir (in China). A significant proportion of patients got antibiotics (42.9\% with $95 \%$ CI $33.3-53.1 \%)$ during their course of illness.

\section{Clinical Outcomes}

Majority of the patients included in the meta-analysis were hospitalized out of which $11.2 \%$ (95\% CI 4.7 $\left.24.2 \%, \mathrm{I}^{2}>75\right)$ received treatment in the intensive care unit. Over $80 \%$ of patients were discharged during the course of the studies ( $83.3 \%$ with $95 \%$ CI $74.6-89.4 \%$ ). The proportion of deaths was $4.2 \%$ (95\% CI $2.6-6.9 \%)$.

Publication Bias and Heterogeneity across Studies

44 out of the 68 variables analyzed did not have significant bias, denoted by an Egger's p value $>0.05$. Majority of the variables were homogenous; only 11 variables had heterogenous data based on $\mathrm{I}^{2}$ index $>$ 75 . 


\section{Discussion}

This meta-analysis showed approximately one-quarter of children were asymptomatic, which is very alarming as these children could be asymptomatic spreaders. Studies reveal comparable or prolonged spread of virus by asymptomatic carriers compared to symptomatic patients ${ }^{11,12}$, however this theory is debatable in recent literature ${ }^{13}$. It could be an important factor to consider in public health policy-making regarding re-opening of schools and play areas. The proportion of asymptomatic patients in China and outside China were similar in our study, $28 \%$ and $21 \%$ respectively. However, these numbers are likely an underestimation due to the small number of data-rich studies published outside China and the sampling bias towards hospitalized and more ill children.

Fever and cough were the most reported symptoms in this meta-analysis, which is consistent with findings reported by two other meta-analyses ${ }^{14}$. This study shows that children present with more upper respiratory findings such as sore throat, nasal congestion and rhinorrhea, whereas dyspnea is less frequently seen in children compared to their adult counterparts ${ }^{15}$. An array of gastrointestinal symptoms such as nausea, vomiting, abdominal pain and diarrhea are reported in pediatric patients with COVID-19, however, it is difficult to discern the proportion of these symptoms attributable to the disease process or part of the sideeffects of therapeutic agents used for treatment or a combination of both. Our study showed approximately one out of seven children had some associated co-morbidity. This was unlike other meta-analyses in the literature which under or infrequently reported comorbidities in pediatric patients ${ }^{13,14}$. We further dissected the comorbidities and found the most common being history of congenital heart disease and obesity. The other co-morbidities associated with COVID-19 presentation in children in our study included history of prematurity, neurological diseases (epilepsy, febrile seizures), asthma, cancer and diabetes, which is distinctive from what was found in most adult studies ${ }^{16}$.

This meta-analysis' findings revealed neutropenia, lymphocytosis and leukopenia as the most common white cell abnormalities. Interestingly, neutropenia was not analyzed in other meta-analyses and hence was not reported $^{14,17}$. This study also showed elevated CRP, LFTs and Lactate Dehydrogenase (LDH) similar to the studies by Ding et al and Zhang el al ${ }^{14,17}$. Our study also showed elevated procalcitonin which was mirrored by Ding et al, but dissimilar from Zhang et al. Co-infections were found in close to onefourth of patients, which is again a peculiar finding of pediatric COVID-19 and the most common coinfections were due to Mycoplasma pneumoniaeand Influenza A/B. Ground glass opacities on CT-scan were the most common radiological finding present in more than a third of patients, resembling previous literature on pediatric COVID-1914. Another interesting radiological finding of this meta-analysis was the equal proportion of unilateral and bilateral lesions in CT-scans in children with COVID-19. In contrast, Mantovani et al reported a higher proportion of unilateral involvement than bilateral, while most other meta-analyses have not described other radio findings other than GGOs ${ }^{13,14,17}$. It is important to note that this study's confidence intervals were narrower and hence more precise than other meta-analyses due to the higher sample size from data-rich articles.

95.9\% of patients were hospitalized and $93.9 \%$ of patients received some form of treatment based on our findings. This observation could be skewed, as most studies with sufficient data to synthesize the clinical findings and outcomes would consist of more symptomatic, sick and hospitalized patients. Anti-virals were the most used therapeutic agents, apart from analgesics and herbal medicines, followed by antibiotics. It is important to note that there was an expected therapeutic variability due to different protocols across the world as well as the changing trends of the pandemic. Intravenous immunoglobulin (IVIG) and glucocorticoids were unique treatment options for pediatric patients with SARS-CoV-2, especially with the emergence of MIS-C ${ }^{18}$. This analysis did not show frequent use of hydroxychloroquine treatment $(5.7 \%)$ as expected and one of the reasons for that could be the evolving therapeutic trends over the course of the pandemic.

Of the hospitalized patients, a small proportion required intensive care admission and even fewer required mechanical ventilation. These important outcomes are under-reported in meta-analysis literature ${ }^{13,14,17}$. Shock was one of the striking complications of the disease course present in about $13 \%$ of patients, as 
highlighted by our meta-analysis. $83.3 \%$ of patients were discharged and this value could be confounded by the time span of the studies and different protocols for patient discharge. Our meta-analysis reports higher than expected death rate $(4.2 \%)$ compared to surveillance data ${ }^{19}$, but this may be due to sampling and reporting bias within studies.

It is possible that our results were confounded by the lack of data outside of China, heterogeneity of a few variables and potential bias within the articles selected. In addition, the variations of diagnostic and therapeutic protocols in different parts of the world and its transformation with the evolving pandemic affects the outcomes reported. Despite the lack of data-rich MIS-C literature published until early July 2020, three MIS-C studies were included in our meta-analysis with 26 patients presenting with prolonged fever and a combination of signs and symptoms such as gastrointestinal manifestations, rash, conjunctivitis, shock with or without multiorgan failure ${ }^{20-22}$. More data on MIS-C is needed to better equip clinicians and epidemiologists in navigating the rough seas of this global pandemic.

\section{Conclusion}

Studies on COVID-19 in children are vital to better understand their unique epidemiological trends, clinical course, laboratory and radiological findings, and prognosis and outcomes. Significant differences exist in all these factors compared to adults. To date, data in children is heavily skewed toward cases from China, and more data is needed from a wider geographic area. This will better prepare us as the cases rise and the dynamics of the pandemic continue to evolve. Emerging information on the proportion of asymptomatic cases and its eventual effect on spread of disease will benefit healthcare providers and public health officers to design appropriate policies, especially with the impending re-opening of schools.

\section{Acknowledgements}

The authors would like to thank Professor Tom Loney for his guidance and Mr. Saad Syed for his early contributions.

\section{References}

1. Naming the coronavirus disease (COVID-19) and the virus that causes it. 2020; https://www.who.int/emergencies/diseases/novel-coronavirus-2019/technical-guidance/naming-thecoronavirus-disease-(covid-2019)-and-the-virus-that-causes-it.

2. WHO. Coronavirus Disease 2019 (COVID-19): Situation Report-208. 2020.

3. WHO Director-General's opening remarks at the media briefing on COVID-19 - 11 March 2020. 2020, 11 March.

4. Cruz AT, Zeichner SL. COVID-19 in Children: Initial Characterization of the Pediatric Disease. Pediatrics. 2020;145(6).

5. Dong Y, Mo X, Hu Y, et al. Epidemiology of COVID-19 Among Children in China. Pediatrics. $2020 ; 145(6)$.

6. Tagarro A, Epalza C, Santos M, et al. Screening and Severity of Coronavirus Disease 2019 (COVID-19) in Children in Madrid, Spain. JAMA Pediatr. 2020.

7. Network CHA. Multisystem Inflammatory Syndrome in Children (MIS-C) Associated with Coronavirus Disease 2019 (COVID-19). 2020.

8. Moher D, Liberati A, Tetzlaff J, Altman DG, Group P. Preferred reporting items for systematic reviews and meta-analyses: the PRISMA statement. PLoS Med. 2009;6(7):e1000097. 
9. Santos WMD, Secoli SR, Puschel VAA. The Joanna Briggs Institute approach for systematic reviews. Rev Lat Am Enfermagem. 2018;26:e3074.

10. Zou L, Ruan F, Huang M, et al. SARS-CoV-2 Viral Load in Upper Respiratory Specimens of Infected Patients. N Engl J Med. 2020;382(12):1177-1179.

11. He X, Lau EHY, Wu P, et al. Temporal dynamics in viral shedding and transmissibility of COVID-19. Nat Med. 2020;26(5):672-675.

12. Maltezou HC, Vorou R, Papadima K, et al. Transmission dynamics of SARS-CoV-2 within families with children in Greece: a study of 23 clusters. J Med Virol. 2020.

13. Zhang L, Peres TG, Silva MVF, Camargos P. What we know so far about Coronavirus Disease 2019 in children: A meta-analysis of 551 laboratory-confirmed cases. Pediatr Pulmonol. 2020;55(8):2115-2127.

14. Grant MC, Geoghegan L, Arbyn M, et al. The prevalence of symptoms in 24,410 adults infected by the novel coronavirus (SARS-CoV-2; COVID-19): A systematic review and meta-analysis of 148 studies from 9 countries. PLoS One. 2020;15(6):e0234765.

15. Yang J, Zheng Y, Gou X, et al. Prevalence of comorbidities and its effects in patients infected with SARS-CoV-2: a systematic review and meta-analysis. Int J Infect Dis. 2020;94:91-95.

16. Ding Y, Yan H, Guo W. Clinical Characteristics of Children With COVID-19: A Meta-Analysis. Front Pediatr. 2020;8:431.

17. Yasuhara J, Kuno T, Takagi H, Sumitomo N. Clinical characteristics of COVID-19 in children: A systematic review. Pediatr Pulmonol. 2020.

18. Team CC-R. Coronavirus Disease 2019 in Children - United States, February 12-April 2, 2020. MMWR Morb Mortal Wkly Rep. 2020;69(14):422-426.

19. Dallan C, Romano F, Siebert J, Politi S, Lacroix L, Sahyoun C. Septic shock presentation in adolescents with COVID-19. Lancet Child Adolesc Health. 2020;4(7):e21-e23.

20. Riphagen S, Gomez X, Gonzalez-Martinez C, Wilkinson N, Theocharis P. Hyperinflammatory shock in children during COVID-19 pandemic. Lancet. 2020;395(10237):1607-1608.

21. Toubiana J, Poirault C, Corsia A, et al. Kawasaki-like multisystem inflammatory syndrome in children during the covid-19 pandemic in Paris, France: prospective observational study. BMJ. 2020;369:m2094.

22. Korkmaz MF, Ture E, Dorum BA, Kilic ZB. The Epidemiological and Clinical Characteristics of 81 Children with COVID-19 in a Pandemic Hospital in Turkey: an Observational Cohort Study. J Korean Med Sci. 2020;35(25):e236.

23. Liu YJ, Chen P, Liu ZS, Li Y, Du H, Xu JL. [Clinical features of asymptomatic or subclinical COVID-19 in children]. Zhongguo Dang Dai Er Ke Za Zhi. 2020;22(6):578-582.

24. Zhang $\mathrm{C}, \mathrm{Gu}$ J, Chen Q, et al. Clinical and epidemiological characteristics of pediatric SARS-CoV-2 infections in China: A multicenter case series. PLoS Med. 2020;17(6):e1003130.

25. Lan L, Xu D, Xia C, Wang S, Yu M, Xu H. Early CT Findings of Coronavirus Disease 2019 (COVID-19) in Asymptomatic Children: A Single-Center Experience. Korean J Radiol. 2020;21(7):919-924.

26. Sun D, Chen X, Li H, et al. SARS-CoV-2 infection in infants under 1 year of age in Wuhan City, China. World J Pediatr. 2020;16(3):260-266.

27. Wu H, Zhu H, Yuan C, et al. Clinical and Immune Features of Hospitalized Pediatric Patients With Coronavirus Disease 2019 (COVID-19) in Wuhan, China. JAMA Netw Open. 2020;3(6):e2010895.

28. Harman K, Verma A, Cook J, et al. Ethnicity and COVID-19 in children with comorbidities. Lancet Child Adolesc Health. 2020;4(7):e24-e25. 
29. Wu HP, Li BF, Chen X, et al. [Clinical features of coronavirus disease 2019 in children aged $<18$ years in Jiangxi, China: an analysis of 23 cases]. Zhongguo Dang Dai Er Ke Za Zhi. 2020;22(5):419-424.

30. Chen J, Wang XF, Zhang PF. [Asymptomatic SARS-CoV-2 infection in children: a clinical analysis of 20 cases]. Zhongguo Dang Dai Er Ke Za Zhi. 2020;22(5):414-418.

31. Li Y, Cao J, Zhang X, Liu G, Wu X, Wu B. Chest CT imaging characteristics of COVID-19 pneumonia in preschool children: a retrospective study. BMC Pediatr. 2020;20(1):227.

32. Zhong Z, Xie X, Huang W, Zhao W, Yu Q, Liu J. Chest CT findings and clinical features of coronavirus disease 2019 in children. Zhong Nan Da Xue Xue Bao Yi Xue Ban. 2020;45(3):236-242.

33. Lu Y, Li Y, Deng W, et al. Symptomatic Infection is Associated with Prolonged Duration of Viral Shedding in Mild Coronavirus Disease 2019: A Retrospective Study of 110 Children in Wuhan. Pediatr Infect Dis J. 2020;39(7):e95-e99.

34. Ma H, Hu J, Tian J, et al. A single-center, retrospective study of COVID-19 features in children: a descriptive investigation. BMC Med. 2020;18(1):123.

35. Song W, Li J, Zou N, Guan W, Pan J, Xu W. Clinical features of pediatric patients with coronavirus disease (COVID-19). J Clin Virol. 2020;127:104377.

36. Nathan N, Prevost B, Corvol H. Atypical presentation of COVID-19 in young infants. Lancet. 2020;395(10235):1481.

37. Tan YP, Tan BY, Pan J, Wu J, Zeng SZ, Wei HY. Epidemiologic and clinical characteristics of 10 children with coronavirus disease 2019 in Changsha, China. J Clin Virol. 2020;127:104353.

38. Jiang S, Liu P, Xiong G, et al. Coinfection of SARS-CoV-2 and multiple respiratory pathogens in children. Clin Chem Lab Med. 2020;58(7):1160-1161.

39. Shen Q, Guo W, Guo T, et al. Novel coronavirus infection in children outside of Wuhan, China. Pediatr Pulmonol. 2020;55(6):1424-1429.

40. Liu W, Zhang Q, Chen J, et al. Detection of Covid-19 in Children in Early January 2020 in Wuhan, China. N Engl J Med. 2020;382(14):1370-1371.

41. Su L, Ma X, Yu H, et al. The different clinical characteristics of corona virus disease cases between children and their families in China - the character of children with COVID-19. Emerg Microbes Infect. 2020;9(1):707-713.

42. Xu Y, Li X, Zhu B, et al. Characteristics of pediatric SARS-CoV-2 infection and potential evidence for persistent fecal viral shedding. Nat Med. 2020;26(4):502-505.

43. Zhang T, Cui X, Zhao X, et al. Detectable SARS-CoV-2 viral RNA in feces of three children during recovery period of COVID-19 pneumonia. J Med Virol. 2020;92(7):909-914.

44. Lu X, Zhang L, Du H, et al. SARS-CoV-2 Infection in Children. N Engl J Med. 2020;382(17):1663-1665.

45. Liu H, Liu F, Li J, Zhang T, Wang D, Lan W. Clinical and CT imaging features of the COVID-19 pneumonia: Focus on pregnant women and children. J Infect. 2020;80(5):e7-e13.

46. Xia W, Shao J, Guo Y, Peng X, Li Z, Hu D. Clinical and CT features in pediatric patients with COVID-19 infection: Different points from adults. Pediatr Pulmonol. 2020;55(5):1169-1174.

47. Wang D, Ju XL, Xie F, et al. [Clinical analysis of 31 cases of 2019 novel coronavirus infection in children from six provinces (autonomous region) of northern China]. Zhonghua Er Ke Za Zhi. 2020;58(4):269-274.

48. Tan X, Huang J, Zhao F, Zhou Y, Li JQ, Wang XY. [Clinical features of children with SARS-CoV-2 infection: an analysis of 13 cases from Changsha, China]. Zhongguo Dang Dai Er Ke Za Zhi. 2020;22(4):294298. 
49. Xing YH, Ni W, Wu Q, et al. Prolonged viral shedding in feces of pediatric patients with coronavirus disease 2019. J Microbiol Immunol Infect. 2020;53(3):473-480.

50. Qiu H, Wu J, Hong L, Luo Y, Song Q, Chen D. Clinical and epidemiological features of 36 children with coronavirus disease 2019 (COVID-19) in Zhejiang, China: an observational cohort study. Lancet Infect Dis. 2020;20(6):689-696.

51. Sun D, Li H, Lu XX, et al. Clinical features of severe pediatric patients with coronavirus disease 2019 in Wuhan: a single center's observational study. World J Pediatr. 2020;16(3):251-259.

52. Ji LN, Chao S, Wang YJ, et al. Clinical features of pediatric patients with COVID-19: a report of two family cluster cases. World J Pediatr. 2020;16(3):267-270.

53. Li W, Cui H, Li K, Fang Y, Li S. Chest computed tomography in children with COVID-19 respiratory infection. Pediatr Radiol. 2020;50(6):796-799.

54. Hu Z, Song C, Xu C, et al. Clinical characteristics of 24 asymptomatic infections with COVID-19 screened among close contacts in Nanjing, China. Sci China Life Sci. 2020;63(5):706-711.

55. Cai J, Xu J, Lin D, et al. A Case Series of children with 2019 novel coronavirus infection: clinical and epidemiological features. Clin Infect Dis. 2020.

\section{Hosted file}

Main Document Tables.docx available at https://authorea.com/users/352580/articles/476753covid-19-under-19-a-meta-analysis

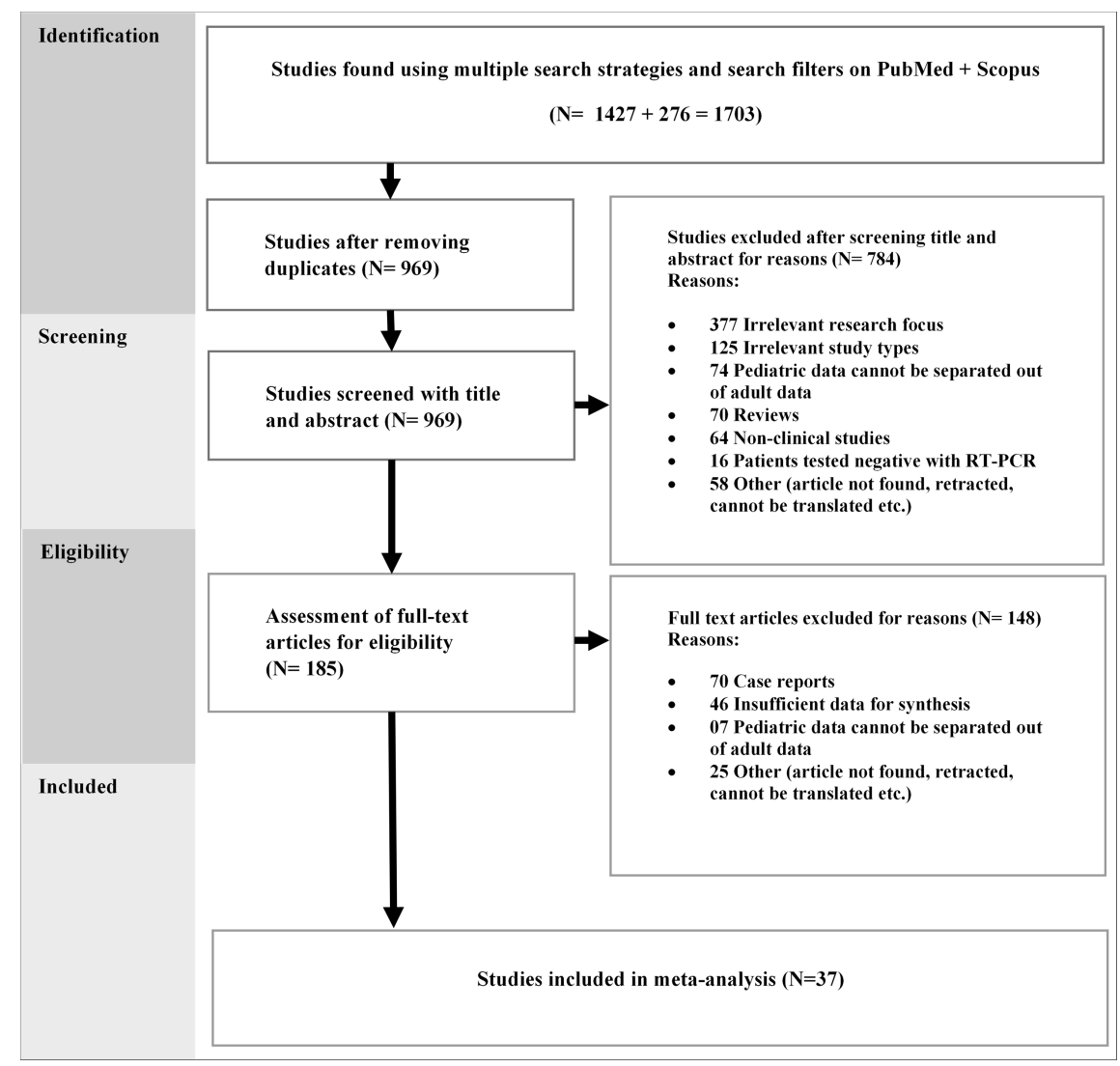

